\title{
Tethered particle motion mediated by scattering from gold nanoparticles and darkfield microscopy
}

\author{
Heidelinde R. C. Dietrich, ${ }^{a}$ Bernd Rieger, ${ }^{a}$ Frank G. M. Wiertz, \\ Frederik H. de Groote, ${ }^{b}$ \\ Hendrik A. Heering, ${ }^{b}$ Ian T. Young, ${ }^{a}$ and Yuval Garini ${ }^{a, c}$ \\ ${ }^{a}$ Department of Imaging Science and Technology, Delft University of Technology, \\ Lorentzweg 1, 2628 CJ Delft, The Netherlands \\ h.r.c.dietrich@tudelft.nl \\ b.rieger@tudelft.nl \\ i.t.young@tudelft.nl \\ ${ }^{b}$ Leiden Institute of Chemistry, Leiden University, Einsteinweg 55, 2333 CC Leiden, The \\ Netherlands \\ f.h.degroote@.chem.leidenuniv.nl \\ f.wiertz@.chem.leidenuniv.nl \\ h.a.heering@chem.leidenuniv.nl \\ ${ }^{c}$ Department of Physics and Institute of Nanotechnology, Bar-Ilan University, Ramat Gan \\ 52900, Israel \\ gariniy@mail.biu.ac.il
}

\begin{abstract}
We measured light scattered from gold nanoparticles with darkfield microscopy in order to perform single molecule detection based on tethered particle motion (TPM). This combination results in a signal to noise ratio of about $40 \mathrm{~dB}$, which allowed us to use $80 \mathrm{~nm}$ diameter gold particles as reporters instead of the typically used polystyrene particles whose sizes are up to $1 \mu \mathrm{m}$. The particle size is crucial in TPM experiments as it can induce a volume-exclusion effect, which results in a stretching force acting on the DNA tether. This affects both the biophysical and statistical properties of the anchored DNA and hence the interpretation of the experimental data. We demonstrated that the gold nanoparticles and darkfield microscopy can be used to characterize the confined Brownian motion of dsDNA-tethered gold particles with a spatial precision of $3 \mathrm{~nm}$. Physical parameters such as the spring constant of the tethered DNA fragment and the persistence length can be derived from the two dimensional (2D) (x, y) projected image data. We have applied this method to various $\mathrm{MgCl}_{2}$ and glycerol concentrations as a proof of principle.
\end{abstract}

Keywords: tethered particle motion, gold nanoparticles, darkfield microscopy, scattering.

\section{INTRODUCTION}

The detection and manipulation of individual biological molecules (e.g. DNA, proteins) has become feasible with the introduction of various single molecule techniques. These techniques are continuously evolving and are based on a range of different principles [14]. Conventional biochemical assays are based on bulk measurements and the data obtained are averaged over a large ensemble of molecules. This does not always permit the understanding of individual molecular mechanisms and spontaneous (equilibrium) reactions cannot be monitored. Single-molecule detection enables the observation of the dynamic behaviour of individual molecules. Hence, single-molecule techniques are an essential tool to obtain fundamental information on biological processes and to a better understanding of the cellular function $[5,6]$.

Various techniques can be applied for the characterization of nucleic acids and DNAprotein interactions [4, 7-11]. One of the most straightforward techniques is tethered particle motion (TPM). It allows the study of biomolecules and biomolecular interactions in a fast and relatively easy way while retaining single molecule character. The achievement of TPM lies in its simplicity [12]. It allows one to look at many interactions 
simultaneously while maintaining single-molecule characteristics. Unlike tweezers and hydrodynamic flow experiments, the DNA/RNA fragments are not stretched, leaving these macromolecules unaltered.

In TPM, a biopolymer, like DNA or RNA is immobilized onto a solid support (e.g. a glass cover slip) at one end and a micrometer or nanometre sized particle at the other end. In solution, the tethered biopolymer and particle are moving due to constrained Brownian motion. The position of the tethered sphere is limited to a certain volume (sometimes called excursion) depending on the size and shape of the immobilized tether. Many enzymes directly interact with DNA and RNA in various biological processes, which very often result in a change in the biopolymer's structure. Thus, the excursion of the particle varies depending on the manner of deformation of the DNA, e.g. DNA looping or DNA extension. The interpretation of the temporal and spatial changes in the position of the reporter particle - which we shall call the radius measured in a 2D coordinate system results in information that allows one to describe the kinetics and biochemical dynamics of the interactions.

Schafer et al. were the first to report on TPM using video-enhanced differential interference contrast microscopy (DIC). They utilized $\varnothing=40 \mathrm{~nm}$ gold particles as reporters and studied the transcription of RNA [13]. More publications followed in which different enzymes were studied by means of video-enhanced DIC microscopy, TIRF microscopy, fluorescence microscopy or conventional transmission microscopy [14-21]. In all of these studies rather large polystyrene/latex particles (with diameters between 200 and $1000 \mathrm{~nm}$ ) were applied.

We have adapted some of the classical TPM characteristics in order to realize a less complex procedure: darkfield, tethered particle motion (DF-TPM). Our method makes use of $\varnothing=80 \mathrm{~nm}$ gold particles as reporters. These particles scatter light and that allows us to use darkfield microscopy, which is straightforward and inexpensive. This combination results in a very strong, non-photobleachable signal, allowing us to monitor biochemical reactions over long time periods $(>1 \mathrm{~h})$ while observing few particles simultaneously thereby reducing the time it takes to measure statistically significant data. A metal bead scatters light with an intensity

$$
I \propto \frac{r^{6}}{\lambda^{4}}\left|\frac{m^{2}-1}{m^{2}+2}\right|^{2},
$$

where $r$ is the bead radius, $\lambda$ is the light wavelength, and $m$ is the ratio of the metal bead index of refraction and the medium index of refraction. As $m$ is about 1.7 for a $80 \mathrm{~nm}$ gold particle, the $m$-dependent part for metal in water is on the order of unity. It is much lower for transparent materials. The scattering intensity for metal beads is three orders of magnitude larger than for a similar sized polystyrene bead [22].

Metal nanoparticles can be detected in various ways due to their scattering, reflection and even fluorescence. Sandoghdar et al. developed a method to detect $5 \mathrm{~nm}$ small nanoparticles by observing the interference of the scattered and reflected light [23]. In this method, the interference results in a dark spot over a bright background. Zheng et al. showed that very small clusters of metal atoms can even fluoresce at wavelengths that can be tuned across the spectrum [24].

Darkfield microscopy was used for various single molecule applications. In darkfield microscopy the sample is illuminated at large angles relative to the optical axis, but the light from the sample is collected only through a cone around the optical axis with a smaller angle. In such an optical setup, light that is reflected directly from the surface is not detected at all, and therefore the background of the whole image is dark. On the other hand, if a particle is scattering light, then the light is usually scattered to all possible directions ( $4 \pi$ radians), and it will be observed. The method allows one to detect scattering particles with very high signal to noise ratio (SNR). It has to be mentioned that due to the kind of illumination and detection of light in darkfield microscopy no interference between the scattered light of the gold particle and the reflected light from the gold surface will occur. Initial attempts to use darkfield microscopy and gold particles was demonstrated by Braslavsky et al. [25]. They showed that back-scattered light of 
various gold particle sizes could be detected by a laser-illuminated, objective-type darkfield microscope. This method is straightforward but suffered from a low SNR, which forced them to use a $290 \mathrm{~nm}$ gold particle for their TPM measurement. Raschke et al. used a variation of darkfield with metal nanoparticles for biomolecular recognition [26]. In this work, small variations in the scattered spectrum were used to detect various molecular bindings to a gold nanoparticle.

Kim et al. demonstrated the usage of darkfield detection with a rather large bead $(>2$ $\mu \mathrm{m})$ for stretching DNA or RNA with flow and detecting enzymatic activity [27]. The scattering from metal beads was so intense that it could even be used for high speed measurements as was shown for studying the flagella rotary motor at frequencies as high as $300 \mathrm{~Hz}$ [28].

In this article, we describe a TPM set-up using a commercial objective-type darkfield microscope and $80 \mathrm{~nm}$ gold particles as reporters (DF-TPM). This method allows us to determine not only the radial mobility of the gold particle but also parameters such as the persistence length and spring constant from the acquired data. We further point out the effect of motion blur on the measurement data. Our method has been applied under different solution conditions.

\section{MATERIALS AND METHODS}

All materials were bought from Sigma-Aldrich, NL unless otherwise stated.

\section{1 dsDNA fragment preparation}

The dsDNA fragments were synthesized from an unmethylated lambda DNA (Promega, NL) template using Expand Long Template PCR System (Roche, NL). dNTPs were bought from Promega, NL. The following primers (Isogen, NL) were used for the production of the 4882 bp: 5'-Biotin-ATA GGC CAG TCA ACC AGC AGG-3' (forward), 5'-disulfide-ATA GGT AAA GCG CCA CGC TCC-3' (reverse). The PCR product was purified according to the QIAquick PCR purification kit manual (Qiagen, NL).

\subsection{TPM sample preparation}

All steps were carried out in a humidified environment (= Petri dish containing a wet paper towel). Passivation of the gold support can be done in two ways: 1. Usage of a mercapto-self-assembled monolayer (m-SAM), e.g. mercaptoundecanoic acid, followed by incubation with a biotin solution; 2 . No SAM, only a biotin/bovine serum albumin solution is used. The choice of the m-SAM depends on the nature of the experiment in order to prevent sticking of the DNA tether and gold nanoparticle. The concentration lies typically around $1 \mathrm{mM}$. The incubation time depends on the length of the m-SAM. We applied an incubation time of about one hour for mercaptohexanol. m-SAM's with a higher number of carbon atoms (e.g. $\mathrm{C}_{11}$ ) should be incubated overnight.

The biotin solution should not exceed $1 \mathrm{mg} / \mathrm{ml}$ as this causes specific sticking of the anti-biotin labelled gold particles. TPM samples were prepared as follows:

Protocol $A$ ( $\mathrm{MgCl}_{2}$ measurements):

The gold support (Arrandee, GER) was incubated with $1 \mathrm{mM}$ mercaptoundecanoic acid solution (in EtOH absolute) over night. The gold slides were then placed into a fresh ethanol solution in order to wash off unbound mercaptoundecanoic acid for about 60 minutes. $0.5 \mathrm{mg} / \mathrm{ml}$ biotin in TE buffer (Tris-HCl $10 \mathrm{mM}$, EDTA $1 \mathrm{mM}$; pH 8.0 at $21^{\circ} \mathrm{C}$ ) was applied onto a small region of the slide and incubated for about 60 minutes and subsequently washed with TE buffer. The purified dsDNA was diluted to a concentration of approximately $10 \mathrm{ng} / \mu \mathrm{l}$ in TE buffer, applied onto the slide and incubated for about 36 $\mathrm{h}$ at $4{ }^{\circ} \mathrm{C}$. Unbound material was washed away by thorough rinsing with TE buffer. Further steps were executed as explained in paragraph: Collective preparation protocol. 
Protocol B (glycerol measurements):

The gold support (Arrandee, GER) was incubated with a passivation solution of 0.5 $\mathrm{mg} / \mathrm{ml}$ biotin and $10 \mathrm{mg} / \mathrm{ml} \mathrm{BSA} \mathrm{(New} \mathrm{England} \mathrm{Biolabs,} \mathrm{UK)} \mathrm{in} \mathrm{TE} \mathrm{buffer} \mathrm{(Tris-HCl} 10$ $\mathrm{mM}$, EDTA $1 \mathrm{mM}$; pH 8.0 at $21^{\circ} \mathrm{C}$ ) for about 60 minutes and subsequently washed with TE buffer. The purified dsDNA was diluted to a concentration of approximately $10 \mathrm{ng} / \mu \mathrm{l}$ in TE buffer, applied onto the slide and incubated over night at $4{ }^{\circ} \mathrm{C}$. Unbound material was washed away by thorough rinsing with TE buffer.

\section{Collective preparation protocol:}

The following steps were applied for both TPM preparation protocols. Gold particles coated with anti-biotin antibodies $(\varnothing=80 \mathrm{~nm}$, Invitrogen, NL and British Biocell, UK) in TE buffer (1:500 dilution) were deposited onto the chip surface and incubated for $1 \frac{1}{2} \mathrm{~h}$ in a humidified Petri dish. Unbound particles were washed off with TE buffer. The prepared gold slides were kept wet by TE buffer until usage. A prepared slide was placed on the lower part of the chip holder and was subsequently closed with a cover slip and filled with TE buffer. This allowed a prolonged observation of the sample over several hours.

The chip holder consists of plastic and has an opening in the middle for the gold chip (Fig. 1). This opening is connected to two syringes allowing the exchange of fluid. A conventional cover slip (thickness $=0.17 \mathrm{~mm}$ ) is used as a cover for the chip holder.

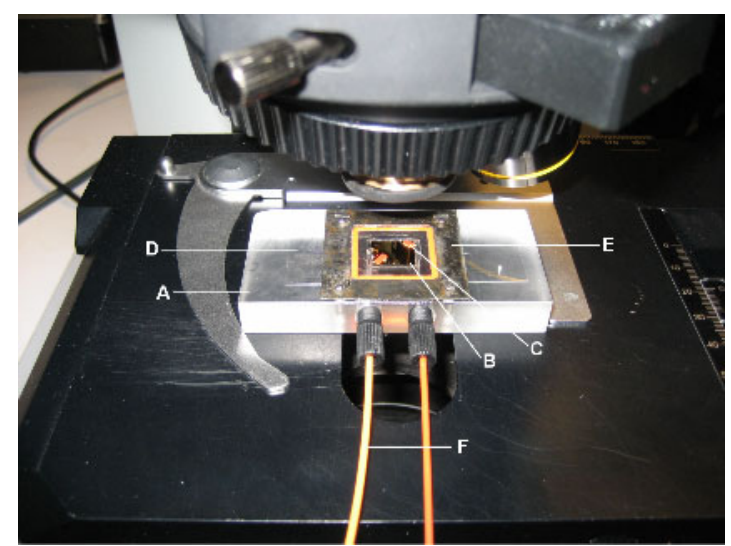

Fig. 1. Chip holder. The chip holder (A) is made out of plastic and has a well in the centre for the placement of the gold support (B) (Arrandee, Germany). This support is held in place by two small rubber tubes $(\mathbf{C})$ that are placed on top of it. A conventional cover slip $(\mathbf{D})($ thickness $=0.17 \mu \mathrm{m})$ is used to close off the space left above the gold support forming a chamber with a volume of approximately $60 \mu$ l. The cover slip is pushed down by a square metal sheet $(\mathbf{E})$, which is tightened by four screws. The holder is connected to two syringes by two rubber tubes $(\mathbf{F})$. This allows an easy exchange of fluid.

\subsection{Solution conditions}

$\mathrm{MgCl}_{2}$ solutions were made in $20 \mathrm{mM}$ Tris- $\mathrm{HCl}$ buffer $\mathrm{pH} 8.0$ at $21^{\circ} \mathrm{C}$. Glycerol solutions were made in TE buffer as weight per volume concentrations.

\subsection{Data collection and data analysis}

The chip holder was placed on a darkfield microscope (BX51, Olympus, NL). A region of interest (ROI) containing reference and moving particles was acquired with a 12-bit CCD camera (Hamamatsu, Orca-ER C4742-95). Illumination was from a $100 \mathrm{~W}$ quartz-halogen source. The sampling densities in $\mathrm{x}$ - and $\mathrm{y}$-directions were: $\Delta \mathrm{x}=0.13 \mu \mathrm{m}$ and $\Delta \mathrm{y}=0.13$ $\mu \mathrm{m}$ using a $50 \mathrm{x} / 0.8$ objective. The CCD camera and the image acquisition are controlled by using SimplePCI (Compix Inc. Imaging Systems, NL). The image and data analysis software was written in MATLAB using our in-house software package DIPimage (a scientific image processing toolbox for MATLAB, Delft University of Technology, The 
Netherlands, http://www.diplib.org). The acquisition frame rate was mainly dependent on the exposure time (50-83 $\mathrm{ms}$ ) and on the size of the region-of-interest (ROI) which was between 12 and 20 frames/s.

From the selected ROI in the first time frame of the image sequence, stationary and moving particles are roughly indicated by hand via a user interface. The remaining procedure is fully automated. The positions of all selected particles are tracked by means of centre-of-mass estimation, which is precise to sub-pixel level for high SNR data of blob-like objects $[29,30]$. Based on the relative averaged motion of stationary marked particles, the global drift is computed. Drift occurs due to translation of the gold support within the opening of the chip holder (electronic supplementary material). No rotation of the sample plane during the experiments has been found. All data and images presented here are drift corrected. We visually inspect $2 \mathrm{D}$ scatter plots of the positions of moving and stationary particles to verify an isotropic distribution. This indicates that there is no flow in the chamber. This additionally allows us to discriminate "good" tethers from "bad" ones. Particles that are bound to two or more tethers exhibit an anisotropic 2D scatter plot and are omitted from further data analysis according to standard practice in TPM [19].

\section{RESULTS}

Our TPM method makes use of $80 \mathrm{~nm}$ sized gold particles as reporters. These metallic particles have the advantage that they scatter polychromatic and monochromatic light depending on the particle size and the illumination type. The signal is strong and does not suffer from photobleaching [31]. This allows us to monitor biological reactions over very long time periods (hours). The fact that we work with scattering implies the possibility of using darkfield microscopy, which is straightforward and inexpensive. This combination (Fig. 2) implies a SNR of about $40 \mathrm{~dB}$ using exposure times between 50-83 ms (electronic supplementary material).

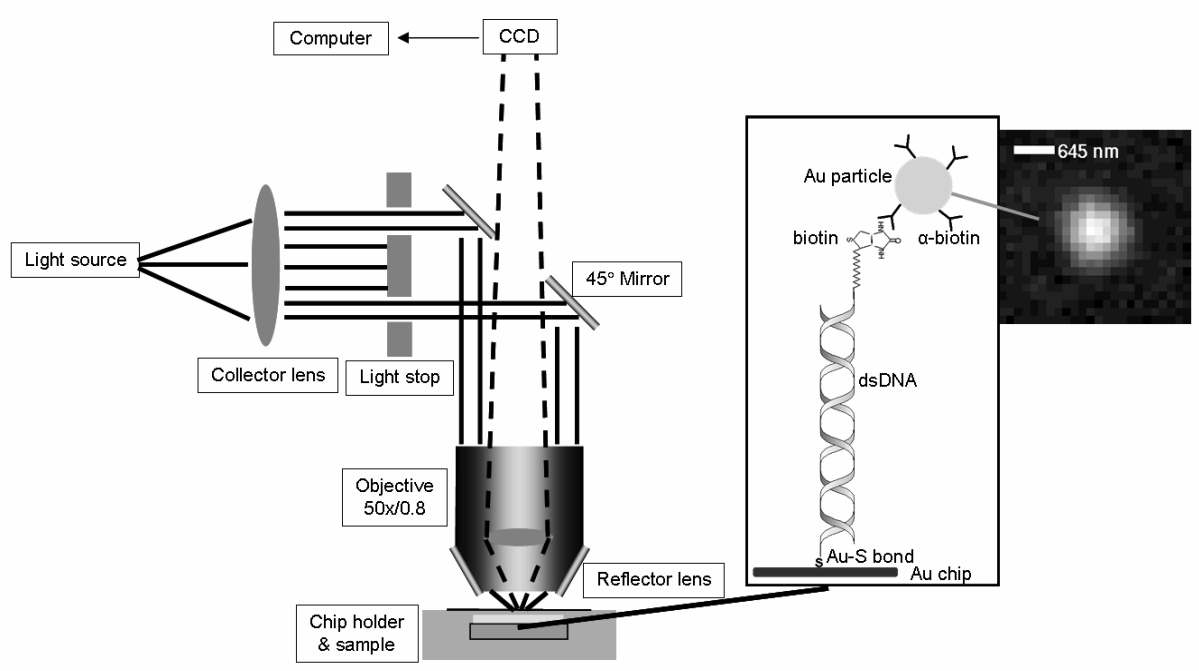

Fig. 2. TPM set-up. Scheme of the darkfield illumination (darkfield microscope, BX51, Olympus, $\mathrm{NL}$ ) of the sample and a close up of the applied chemistry. A 4882 bp dsDNA fragment is tethered to the gold surface by a disulfide group and the $\varnothing=80 \mathrm{~nm}$ gold particle is attached to the fragment by a biotin/anti-biotin antibody interaction. The outermost right picture is an image of such a tethered gold particle. The width of the particle image is about 10 pixels $\approx 1.3 \mu \mathrm{m}$. 
The imaged width of a reporter particle was approximately 10 pixels, which was equivalent to $1.3 \mu \mathrm{m}$ with an intensity of about $10^{4}$ photons/particle/frame. We converted CCD ADUs (analog-to-digital-unit) to photons via a CCD gain calibration [32]. Using a frame rate of 12-20 frames/sec, allows the detection of fast reactions and transitions with rather simple image analysis procedures. Higher frame rates are possible with adequate CCDs. The position precision (= statistical error) with which the gold particle could be determined in the $(x, y)$ plane was approximately $\sigma=3 \mathrm{~nm}$ (electronic supplementary material). Ober et al. have shown that the theoretical position precision of an optical system equipped with a CCD camera and setting comparable to ours is better than $1 \mathrm{~nm}$ [30]. This high-precision results from the good SNR due to the intense light scattering of the particle and the low background from the flat gold support [30]. It emphasizes the advantages of the presented technique.

Segall et al. pointed out that large particles have a significant influence on the motion of the DNA/RNA tether in solution due to steric hindrance [33]. They indicate that the bead can alter the conformation of the tether and the statistical properties of the attached biopolymer, leading to an interpretation of the resulting data that is influenced by a volume-exclusion effect that occurs when there is proximity of the bead to the surface. This effect results in an entropic upwards stretching-force on the DNA/RNA tether and therefore depends on the bead size and the tether length. Segall et al. defined a dimensionless number $\left(N_{R}\right)$ that allows one to make a prediction as to whether the detected constrained Brownian motion suffers from a particle-induced volume-exclusion effect. Detailed analysis of the particle size with respect to the contour length and persistence length shows that this effect has negligible influence when $N_{R} \equiv R / \sqrt{L \xi / 3}$ is smaller than one, where $R$ is the radius of the bead, $L$ is the contour length and $\xi$ is the persistence length of the biopolymer. The application of this equation to our experimental set-up, with a dsDNA contour length of $1660 \mathrm{~nm}$, a persistence length of $50 \mathrm{~nm} \mathrm{[34]} \mathrm{and} \mathrm{a}$ bead radius of $40 \mathrm{~nm}$, results in an excursion number of 0.24 . According to Segall's theory, steric hindrance does not significantly affect the conformation space in our experiment.

\subsection{D (x, y) projected data}

A typical $x, y$-scatter plot and an excursion histogram of a moving, tethered $4882 \mathrm{bp}$ dsDNA fragment are shown in Fig. 3a, b. The excursion histogram can be fit to the expected end-to-end distance distribution of the DNA. This can be calculated with the ideal chain model. According to the model, the two dimensional distribution of the endto-end distance is given by [35]:

$$
P_{2 D}(R) 2 \pi R d R=\frac{3}{4 \pi L P} \exp \left(-\frac{3 R^{2}}{4 L P}\right) 2 \pi R d R,
$$

where $P_{2 D(R)}$ is the probability density for finding the end-to-end distance between $R$ and $R$ $+d R$ in the $x, y$-plane. $L$ is the DNA contour length and $P$ is the persistence length of the DNA. Figure $3 \mathrm{~b}$ also shows the best fit of the ideal chain model to the experimental data with a single parameter (the persistence length) assuming that the DNA length is 1660 $\mathrm{nm}$. The extracted persistence length was $50 \pm 7 \mathrm{~nm}$. 

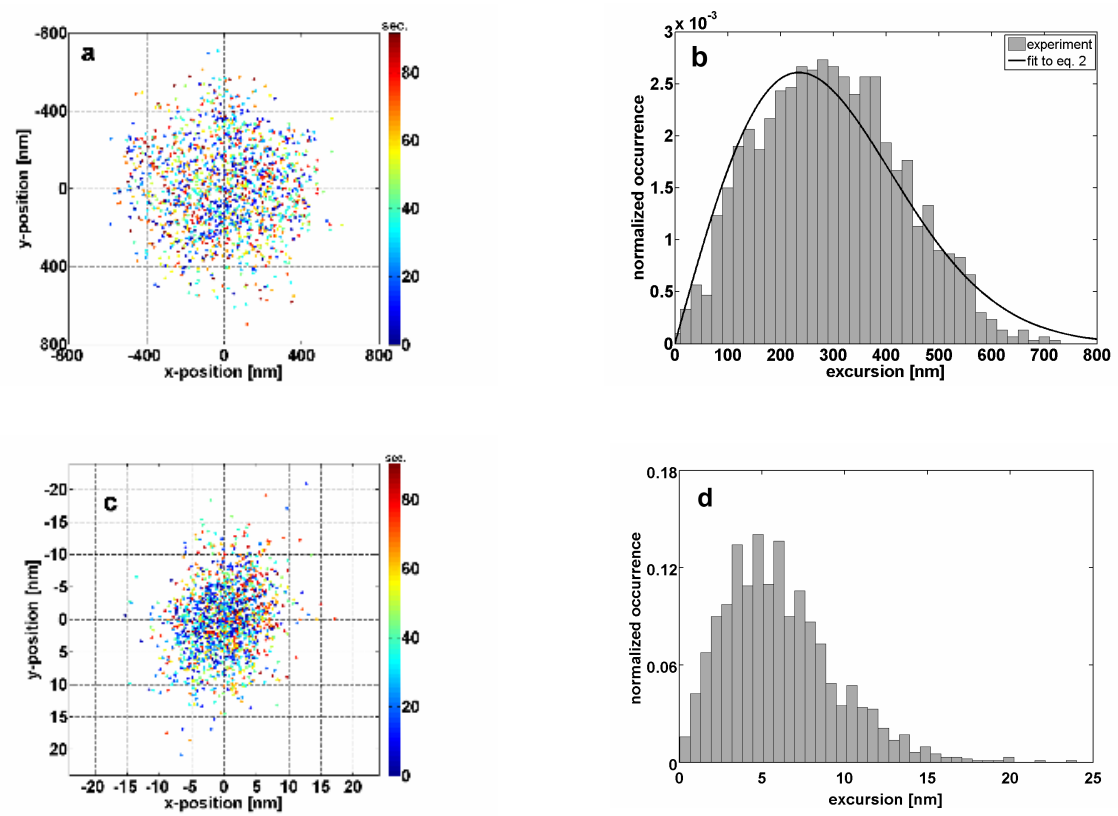

Fig. 3. $x, y$-scatter plots and excursion histograms of a moving tethered particle and a stationary particle (drift corrected). The time course (in seconds) of the tethered particle is indicated by the colour. The exposure time was $60 \mathrm{~ms}$. (a) A typical $x, y$-scatter plot of a tethered $4882 \mathrm{bp}$ dsDNA fragment labelled with a $\varnothing=80 \mathrm{~nm}$ gold particle. The excursion of the particle is defined as the distance the particle travels in 2D depending on the DNA tether length. The mean excursion of such a particle is $297 \mathrm{~nm}$ when measured over 1500 frames. (b) The radial histogram of such a tethered gold particle. The line represents a single parameter fit of an ideal chain model (Rayleigh distribution in $r)$ to the experimental data $\left(\sigma_{r}=235 \mathrm{~nm} ; r^{2}=0.94\right)$. The extracted persistence length is about $50 \pm 7 \mathrm{~nm}$. (c) The $x, y$-scatter plot of a stationary reference particle. The mean excursion is $6 \mathrm{~nm}$ (1500 frames). This value represents the control value of our TPM system. It characterizes the robustness of the method. (d) The radial histogram of a stationary particle.

The excursion radius was calculated according to $r=\sqrt{(x-<x>)^{2}+(y-<y>)^{2}}$. The average excursion of such a labelled DNA fragment $\langle r\rangle$ was found to be $297 \mathrm{~nm}$ (for DNA tether shown in Fig. 3a,b). The radial position of the tethered particle shows no favoured direction over 1500 frames. The nominal length of a 4882 bp dsDNA strand is $1660 \mathrm{~nm}$, which is 5.6 times larger than the average radial distance we measured. We found a maximum excursion of about $700 \mathrm{~nm}$. This difference is a natural result of the end-to-end distance statistic for a polymer such as dsDNA and can be described by the worm-like chain model [36]. It is very unlikely that the DNA tether will be stretched to its full contour length without applying external force as is done with magnetic or optical tweezers.

The mean excursion of a stationary reference particle (after drift correction) was $6 \mathrm{~nm}$ (Fig. 3c). The particle was followed typically over 1500 frames. This value sets a criterion on the accuracy of our TPM system and represents the measurement uncertainty of the equipment and analysis all together. The fact that a "stationary" particle moves at all is due to stochastic processes like mechanical vibration of the optical set-up, random noise of the CCD camera and quantization errors in the analysis procedure. This value should not be confused with the position precision of the method. The excursion histogram of a stationary particle is shown in Fig. 3d. 
We have also measured the excursion of a 362 base pairs dsDNA fragment (electronic supplementary material). The mean excursion for this non-stationary particle was $72 \mathrm{~nm}$, which is approximately 12 times larger than that of a stationary particle. In principle, the fragment length could be shortened even further and would not suffer from steric hindrance. One has to keep in mind, however, that the mean excursion has to be distinguishable from a stationary particle and conditions have to be such that the excursion number is smaller than one.

\section{2 dsDNA tether elasticity}

The elasticity of dsDNA at low and intermediate forces can be numerically estimated according to the one-dimensional worm-like chain (WLC) model [36-38] by

$$
\frac{F P}{k_{B} T}=\frac{1}{4}\left(1-\frac{x}{L}\right)^{-2}-\frac{1}{4}+\frac{x}{L}
$$

where $F$ is the stretching force, $P$ is the persistence length, $k_{B}$ is the Boltzmann constant, $T$ is the temperature, $x$ is the extension of the dsDNA and $L$ is its contour length. At very low forces, dsDNA behaves like a Hookean spring [37] and Eq. 3 can be approximated by

$$
F=\frac{3 k_{B} T}{2 P} \frac{x}{L}
$$

The spring constant can therefore be estimated by

$$
k=\frac{3 k_{B} T}{2 P L} .
$$

The application of Boltzmann statistics to the individual $x$ and $y$ displacement distributions according to $P(x)=\frac{1}{Z} e^{-k x^{2} / 2 k_{B} T}$ results in energy profiles of the dsDNA tether [16]. $P(x)$ is the probability of the DNA/particle system being in a certain position, $Z$ is a normalization factor, $k$ is the spring constant, and $x$ is the displacement in the plane.

Fig. 4a,b shows a typical energy profile for the 4882 bp dsDNA fragment we used. The symmetry and the quadratic fit of the profile demonstrate the spring-like behaviour of the dsDNA tether. The spring constant $k$ can be extracted from the quadratic fit to the obtained energy profile. The spring constant of the energy profile depicted in Fig. 4a is $k$ $=0.079 \pm 0.003 \mathrm{pN} / \mu \mathrm{m}$ ( $x$-displacement) and in Fig. $4 \mathrm{~b} k=0.076 \pm 0.003 \mathrm{pN} / \mu \mathrm{m}(y$ displacement). The stated errors are the standard deviation computed from the covariance matrix of the fit. 

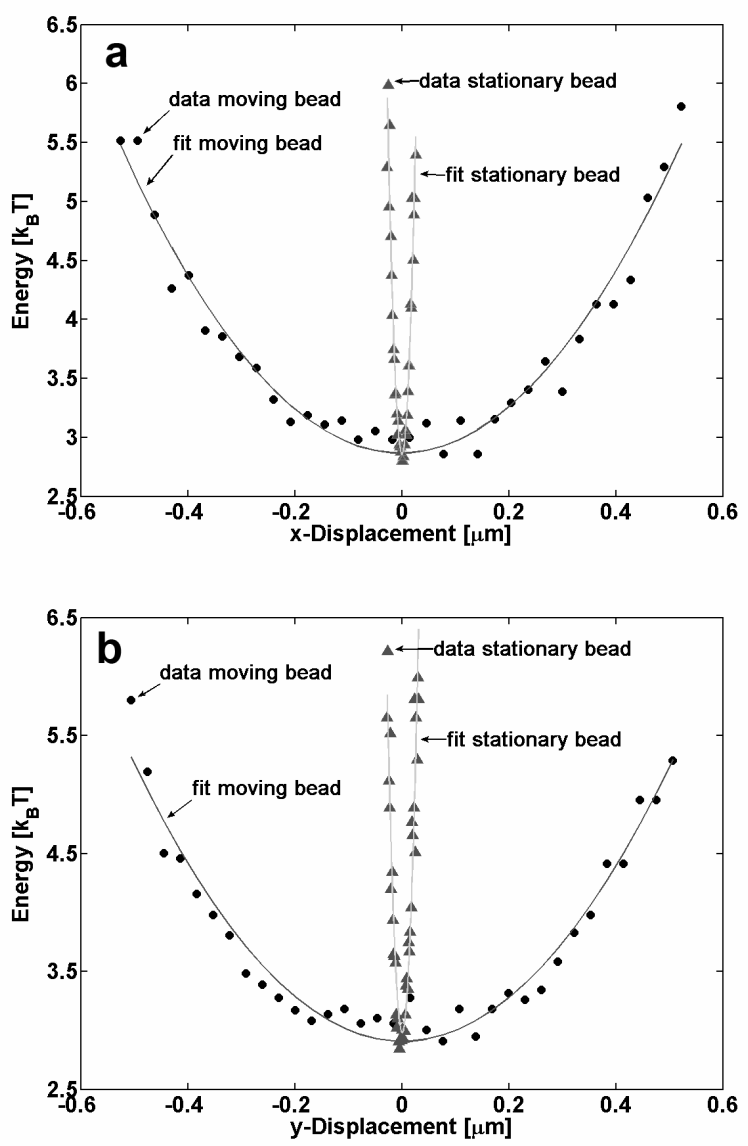

Fig. 4. Energy profiles of a moving and a stationary particle (drift corrected). By applying the Boltzmann distribution, the spring constant of the DNA tether can be estimated from a quadratic fit to the energy profile derived from a lateral displacement histogram. Typical $x, y$-displacement energy profiles of a $4882 \mathrm{bp}$ dsDNA (dots) and a stationary particle (diamonds) are shown. The solid lines depict the quadratic fit to the experimentally measured energy profiles. The exposure time was $50 \mathrm{~ms}$. (a) The energy profile derived from an $x$-displacement histogram. The estimated spring constant was $0.079 \pm 0.003 \mathrm{pN} / \mu \mathrm{m}$ (fit: $r^{2}=0.96$ ). The grey diamonds represent the energy profile of a stationary particle, with a spring constant of $33.5 \pm 1.56 \mathrm{pN} / \mu \mathrm{m}$ (fit: $r^{2}=0.94$ ). (b) The experimentally determined spring constant derived from the energy profile of the $y$-displacement histogram was $0.076 \pm 0.003 \mathrm{pN} / \mu \mathrm{m}$ (fit: $r^{2}=0.95$ ). The spring constant of the stationary particle was $29.8 \pm 1.56 \mathrm{pN} / \mu \mathrm{m}$ (fit: $\left.r^{2}=0.91\right)$.

Averaging over 27 DNA tethers results in a median spring constant of $k=0.11 \pm 0.04$ $\mathrm{pN} / \mu \mathrm{m}$. Blumberg et al. published a value of $0.095 \mathrm{pN} / \mu \mathrm{m}$ (with an average value of 18 tethers of $0.10 \pm 0.01 \mathrm{pN} / \mu \mathrm{m}$ ) by means of TIRF-TPM [16]. The theoretical spring constant can be calculated from Equation 5. Applying this equation for our case, with a DNA contour length of $1660 \mathrm{~nm}$ and a literature-based persistence length of $50 \mathrm{~nm}$ (= physiological conditions), a value of $k=0.074 \mathrm{pN} / \mu \mathrm{m}$ results. Rewriting Equation 5 as $P=\frac{3 k_{B} T}{2 k L}$ allows us to estimate the lower and upper bounds on the persistence length which in our case means that, when $k=0.11 \pm 0.04 \mathrm{pN} / \mu \mathrm{m}$, we calculate a persistence length of $25 \leq 35 \leq 56 \mathrm{~nm}$. These values of $k$ and $P$ are consistent with the theory and the experimental results reported in the literature [34, 37, 39-42].

One way to view DF-TPM is in terms of the rms force felt by the DNA/bead system that is $\sqrt{\left\langle F^{2}\right\rangle}=k \sqrt{\left\langle r^{2}\right\rangle}=0.032 \mathrm{pN}$ at room temperature. 


\subsection{Influence of $\mathrm{MgCl}_{2}$ on the dynamic excursion and spring constant}

The conformation of DNA changes in the presence of multivalent cations [43]. This effect might be due to charge screening, decreasing repulsion between DNA segments or due to bending and/or distortion of the DNA structure. We measured the effect of $\mathrm{MgCl}_{2}$ on the excursion and the spring constant of the DNA tether using DF-TPM. The mean excursion, spring constant and potential energies for all concentrations are shown in Table 1.

Table 1. Influence of $\mathrm{MgCl}_{2}$ on the spring constant and the excursion of a tethered dsDNA fragment.

\begin{tabular}{|c|c|c|c|c|}
\hline & \multicolumn{4}{|c|}{$\mathrm{MgCl}_{2}$} \\
\hline Concentration $^{\dagger}$ & $0 \mathrm{mM}$ & $2.5 \mathrm{mM}$ & $5 \mathrm{mM}$ & $10 \mathrm{mM}$ \\
\hline $\begin{array}{l}\text { mean excursion } \\
\pm \mathrm{std}^{\ddagger} \\
{[\mathrm{nm}]<r>}\end{array}$ & $267 \pm 55$ & $212 \pm 48$ & $197 \pm 47$ & $111 \pm 46$ \\
\hline $\begin{array}{l}\text { median spring } \\
\text { constant } \pm \text { std } \\
{[\mathrm{pN} / \mu \mathrm{m}] \boldsymbol{k}_{\text {med }}}\end{array}$ & $0.11 \pm 0.04$ & $0.14 \pm 0.3$ & $0.20 \pm 0.09$ & $0.40 \pm 0.21$ \\
\hline 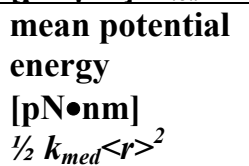 & 3.9 & 3.2 & 3.9 & 2.5 \\
\hline $\begin{array}{l}\text { persistence } \\
\text { length bounds* } \\
\text { [nm] }\end{array}$ & $25 \leq 35 \leq 56$ & $\mathrm{n} / \mathrm{a} \leq 8 \leq 27$ & $13 \leq 19 \leq 36$ & $6 \leq 9 \leq 20$ \\
\hline
\end{tabular}

We observed an overall decrease in mean excursion in the presence of various $\mathrm{MgCl}_{2}$ concentrations. For each concentration at least ten independent measurements were taken. The addition of $2.5 \mathrm{mM}, 5 \mathrm{mM}$, and $10 \mathrm{mM} \mathrm{MgCl}_{2}$ led to a reduction in mean excursion of $21 \%, 26 \%$, and $59 \%$. The median spring constant increased to $0.14 \pm 0.3 \mathrm{pN} / \mu \mathrm{m}, 0.20$ $\pm 0.09 \mathrm{pN} / \mu \mathrm{m}$ and $0.40 \pm 0.21 \mathrm{pN} / \mu \mathrm{m}$ compared to $0.11 \pm 0.04 \mathrm{pN} / \mu \mathrm{m}$ of the dsDNA fragment under the condition where no divalent cation was added ${ }^{\S}$. Further, the potential energy of the DNA/particle system could be calculated from the experimentally determined spring constant and the mean excursion. The persistence length was estimated as mentioned before and was $25 \leq 35 \leq 56 \mathrm{~nm}, \mathrm{n} / \mathrm{a} \leq 8 \leq 27 \mathrm{~nm}, 13 \leq 19 \leq 36 \mathrm{~nm}$, and $6 \leq 9 \leq 20 \mathrm{~nm}\left(0 \mathrm{mM}\right.$ to $\left.10 \mathrm{mM} \mathrm{Mg}^{2+}\right)$. The large errors with 2.5 and $10 \mathrm{mM} \mathrm{Mg}^{2+}$ are due the small number of particles that passed standard evaluation of the isotropy of the 2D scatter plots and were hence included for calculations. The lower bound of the persistence length for $2.5 \mathrm{mM}$ was not specified as it represents a negative number using Equation 5 (see footnote concerning $k$ ).

\section{Viscosity and its influence on tethered particle motion}

Glycerol $\left(\mathrm{C}_{3} \mathrm{H}_{5}(\mathrm{OH})_{3}\right)$ is a very viscous sugar alcohol, which makes it ideal for studying the influence of the viscosity on the confined motion of the DNA tether. We examined changes in the motion of the DNA/particle system as a function of the glycerol viscosity.

The motion was calculated using the "diffusion" of the particle according to $D=\frac{<v^{2}>\Delta t}{2 n}$ where $v^{2}$ is defined as $\left\langle x^{2}>/ \Delta t^{2}, \Delta t\right.$ is the exposure time and $n$ corresponds

\footnotetext{
${ }^{\dagger}$ number of particles used: $0 \mathrm{mM}=27 ; 2.5 \mathrm{mM}=6 ; 5 \mathrm{mM}=7 ; 10 \mathrm{mM}=6$;

${ }^{\ddagger}$ Errors are the standard deviation (std) of the individual measurement.

*The upper and lower bounds on the persistence length using Equation 5 and the range of $k$ given above are specified as $\leq$.

${ }^{\S}$ The value for $k$ associated with $2.5 \mathrm{mM}$ has a large std due to a small sample size $(\mathrm{N}=$ 6 ). It is, of course, not physical possible for $k$ to be less than or equal to zero.
} 
to the observed spatial dimensions (here $n=2)$. The velocity was calculated via the $2 \mathrm{D}(x$, $y)$ projected data. The determined values of the confined motion at various glycerol viscosities are depicted in Table 2.

Table 2. Experimental values of the confined motion of the tethered particle and theoretical diffusion constants for freely diffusing particles for various glycerol viscosities. ${ }^{* *}$

\begin{tabular}{lll}
\hline $\begin{array}{l}\text { Glycerol \% (w/v) } \\
\text { Viscosity [mPas] }\end{array}$ & $\begin{array}{l}\text { Experimental value for } \\
\text { confined motion } \\
{\left[\boldsymbol{\mu m}^{2} / \mathbf{s}\right]}\end{array}$ & $\begin{array}{l}\text { Stokes-Einstein for freely } \\
\text { diffusing particle } \\
\mathbf{D}\left[\boldsymbol{\mu m}^{2} / \mathbf{s}\right]\end{array}$ \\
\hline 0 & $0.42 \pm 0.07$ & 5.46 \\
1.005 & $0.39 \pm 0.07$ & 4.19 \\
\hline 10 & & \\
1.31 & $0.33 \pm 0.10$ & 2.2 \\
\hline 30 & & \\
\hline 2.5 & $0.25 \pm 0.05$ & 0.92 \\
\hline 50 & & \\
\hline 6 & $0.18 \pm 0.03$ & 0.51 \\
\hline 60 & & \\
\hline
\end{tabular}

A change in the confined motion as a function of varying dynamic viscosity could be detected. The motion decreased from the $0 \%(\mathrm{w} / \mathrm{v})$ glycerol solution to the $60 \%(\mathrm{w} / \mathrm{v})$ glycerol solution by $57 \%$. The theoretical diffusion constant (Table 2) was calculated according to the Stokes-Einstein relation $D=\frac{k_{B} T}{6 \pi \eta R}$ where $\eta$ is the dynamic viscosity and $R$ is the radius of the particle and provides a frame-of-reference for our measurements.

\section{DISCUSSION}

In this article, we describe a new TPM technique. Our method (DF-TPM) is based on darkfield microscopy performed with a CCD camera. We make use of $80 \mathrm{~nm}$ large gold particles as reporter molecules. The combination of these gold particles and darkfield microscopy results in a high SNR ob about $40 \mathrm{~dB}$, which is slightly reduced by a factor of 1.2 applying an exposure time of $10 \mathrm{~ms}$ as reported by Brinkers et al. [45]. Additionally, the small particle size minimizes the distortion of the DNA conformation by an entropic stretching force, which results from the particle's proximity to the surface of the solid support [33, 45]. This is important as the obtained data might otherwise be misinterpreted. The effective stretching force can affect the interaction with other molecules, e.g. in DNA-protein interactions. Segall et al. have shown that the loop formation rate of DNA interacting with lac-repressor protein was underestimated by a factor of two in a publication by Finzi and Gelles [15]. It should be clarified that the ratio of the particle size and tether length are less crucial if the experimental procedure is biochemical in nature and not biophysical. We obtained an excursion number of 0.24 under the conditions described in this publication assuming a persistence length of $50 \mathrm{~nm}$. The apparent persistence length in our measurements, however, is about $35 \mathrm{~nm}$. When we use this value, an excursion number of 0.29 results. The persistence length could be theoretically reduced to about $3 \mathrm{~nm}$ (same contour length and particle radius) and still retain an excursion number less than one. Further, we can, in principle, shorten the dsDNA fragment to 285 bp using the $80 \mathrm{~nm}$ large gold particle and still be able to detect constrained Brownian

\footnotetext{
${ }^{* *}$ Glycerol viscosities were taken from Ref. 44 . at $20^{\circ} \mathrm{C}$. The mean value and the standard deviation of a least 10 independent measurements per concentration are shown.
} 
motion without a significant volume-exclusion effect between the particle and the chip surface.

Only Schafer et al. have used small gold particles, but that was performed in combination with DIC microscopy [13]. Yet, the combination of darkfield microscopy that we developed has significant advantages over DIC microscopy. In darkfield microscopy a single bright spot indicates the particle position, while in DIC microscopy the particle position has to be found as the centre of a derivative-like function (where the intensities are grey) and therefore suffers from a reduced SNR.

The gold support implicates the use of a different surface chemistry compared to a glass support. The DNA fragments were immobilized onto the gold surface via a disulfide group. It is well known that thiol- and disulfide groups self-assemble in a very regular and rapid manner on gold generating a strong covalent gold-sulphur bond $\left(D_{0}=40 \mathrm{kcal} / \mathrm{mol}\right)$ [46]. Self-assembled monolayers (m-SAM) can be applied for surface passivation. mSAMs are available in a variety of different end groups that are either positively charged, negatively charged or uncharged, allowing the tuning of the passivation. A selection of articles on self-assembled monolayers can be found in the reference list [47-50]. Passivation with only biotin/BSA was applied as well. One could argue that in TPM experiments stationary particles (for drift correction) are hard to avoid. We noticed, however, that the number of immobile particles depends on the applied passivation surface. It has therefore to be experimentally determined, which concentrations are useful depending upon the experimental conditions. We generally use three stationary particles for drift correction.

Wong et al. have shown that motion blur affects the statistics of fluctuation measurements if the DNA/RNA tether behaves as a harmonic oscillator [51]. The experimentally determined excursion and diffusion coefficient can therefore be underestimated and the spring constant overestimated for long exposure times. Towels et al. studied the issue of motion blur in TPM experiments [52]. They examined the change in rms excursion as a function of exposure time (between $1 \mathrm{~ms}$ and $500 \mathrm{~ms}$ ). It is obvious from their calibration curve that up to an exposure of $10 \mathrm{~ms}$, their measurements did not much suffer from motion blur (tether length $=901 \mathrm{bp}$; particle $\varnothing=490 \mathrm{~nm}$ ). We performed TPM measurements between 10 and $100 \mathrm{~ms}$ exposure times (dsDNA fragment $2672 \mathrm{bp}$ ) in order to determine the extent of motion blur in our data (electronic supplementary material). The rms excursion decreased by about $29 \%$ from $216 \pm 34 \mathrm{~nm}$ to $153 \pm 28 \mathrm{~nm}$ (10 to $100 \mathrm{~ms})$. The spring constant increased from $0.25 \pm 0.08$ to $0.53 \pm 018$ $\mathrm{pN} / \mu \mathrm{m}(10$ to $100 \mathrm{~ms})$. All data presented in this article, however, were monitored at exposure times between 50 and $80 \mathrm{~ms}$. The apparent spring constant of the dsDNA tether (4882 bp) was $0.11 \pm 0.04 \mathrm{pN} / \mu \mathrm{m}$ and the estimated apparent persistence length $25 \leq 35$ $\leq 56 \mathrm{~nm}$. An explanation for this discrepancy from the reported value of approximately 50 $\mathrm{nm}$ [34] is therefore presumable due to motion blur. The effect of motion blur should be determined every time the bead size, tether length or viscosity of the buffered solution changes as the excursion of the reporter particle depends on these factors. Emphasis should be laid on the fact that motion blur is crucial if quantitative measurements are the goal of the study and less importance for (qualitative) assays. Although our data presented here are biased by motion blur, it is sufficient for the validation of DF-TPM as demonstrated with the $\mathrm{MgCl}_{2}$ and glucose experiments.

Elevated temperatures on the surface of the gold support could be another factor influencing the persistence length. Lu et al. have shown that a maximum persistence length is obtained at $20^{\circ} \mathrm{C}$ [39]. Lower and higher temperatures lead to a decrease of the persistence length of approximately 10 to $15 \%$. Halogen light sources produce a considerable amount of heat during operation and the heat could warm the sample during measurement. Although we did not find a significant elevation of the temperature $\left(<1^{\circ} \mathrm{C}\right)$ on the top surface of the gold support, we cannot exclude local heating effects on the gold particle. The temperature was monitored for 30 minutes with temperature recordings every 30 seconds. The measured temperature was $19^{\circ} \mathrm{C}$. Partial sticking of the DNA on an imperfect passivated gold surface could be another explanation for a reduced persistence length. We argue, however, that sticking events would result in a broad spread in the 
average excursion of tethered particles but this was not the case in our measurements where we obtained an average excursion of $267 \pm 55 \mathrm{~nm}(\mathrm{~N}=27$ particles $)$.

We showed that our TPM method can be used to monitor the effect of $\mathrm{MgCl}_{2}$ (Table 1) on the mean excursion and the spring constant of the tethered DNA fragment. The estimated persistence lengths decreased from $35 \mathrm{~nm}\left(0 \mathrm{mM} \mathrm{Mg}^{2+}\right)$ to $9 \mathrm{~nm}\left(10 \mathrm{mM} \mathrm{Mg}^{2+}\right)$. Values between $30 \mathrm{~nm}$ and $70 \mathrm{~nm}$ under various conditions have been reported in literature [39-42, 53]. We have not disregarded the fact that the $\mathrm{Mg}^{+}$could have immobilized the DNA onto the gold surface. This is unlikely as we have shown that the average excursion is consistent over 27 measured tethered particles. The decrease of the estimated persistence length with increasing $\mathrm{Mg}^{2+}$ concentration follows the trend that was predicted theoretically by Odijk and independently by Skolnick and Fixman (OSF theory) [54, 55]. According to the OSF theory, the persistence length is inversely proportional to the salt concentration. Our data is consistent with the characteristics of DNA in salty environments.

The confined motion of the gold particle was studied as a function of glycerol viscosity. The results support our hypothesis that the motion, as we measure it, should decrease with higher solution viscosities. The determination of the dependence of the confined motion on the viscosity was, however, not very sensitive. The experimental values of the confined motion varied by a factor of two from the lowest to the highest glycerol viscosity, which changed by a factor of ten. This discrepancy may be explained by the long exposure times that were between 50 and $80 \mathrm{~ms}$. These were not short enough to overcome the constraint of the tether in order to determine the "free" motion. We calculated that a minimum acquisition time of about $5 \mathrm{~ms}$ should have been applied in order to do so. The calculation is straightforward and given by $\frac{\left\langle x^{2}\right\rangle}{4 D}=t .\left\langle x^{2}\right\rangle$ is the average excursion of a 4882 bp DNA fragment, which was about $0.3 \mu \mathrm{m}$ and $D$ is the diffusion constant of a freely moving particle taken from Table 2 , which was about 5 $\mu \mathrm{m}^{2} / \mathrm{s}$. This may, however, be a fundamental problem as a short acquisition time reduces the SNR and contrast of a digital image. The particle localization accuracy would be compromised. The application of an electron multiplying CCD (EM-CCD) camera may overcome these problems. Another argument for the smaller diffusion coefficients could be reduced hydrodynamic mobility and hence reduced diffusion due to the proximity of the particle to the gold support. Bevan et al. have studied hindered diffusion near a wall [56]. According to their publication we can assume that our TPM measurements did not suffer from such effects taking an elevation height of the sphere of 200 to $250 \mathrm{~nm}$ (from excursion distribution data) and a sphere radius of $40 \mathrm{~nm}$. It is necessary, however, to consider a hindered diffusion every time different fragment lengths are used. Generally in TPM, the motion of the tether/particle system becomes more precise with higher viscosities as can be deduced from Table 2 .

DF-TPM shares one disadvantage with other TPM methods except the TPM-TIRF technique Blumberg et al. published. It does not allow the 3D characterization of the confined Brownian motion. This is, however, not necessary for kinetic assays or the investigation of binding events in general. The TIRF Blumberg method has the advantage of recording the particle's movement in 3D. They used large fluorescent latex particles (200 $\mathrm{nm}$ to $1 \mu \mathrm{m}$ in diameter) and photobleaching can be a limiting factor of this method even if stroboscopic illumination is used. They present no data on how much photobleaching is reduced using stroboscopic illumination. Additionally, the TIRF evanescent field rapidly decreases with distance from the sample surface, resulting in a restricted use of DNA/RNA fragment lengths. For comparison, we performed measurements with $\varnothing=200 \mathrm{~nm}$ large FluoSpheres NeutrAvidin labelled microspheres from Molecular Probes. These spheres have the property that they decay 10,000 times slower than conventional fluorescent probes (product information from Molecular Probes, Cat. no. F20884). These microspheres photobleached within 4 to 5 minutes taking an exposure time of $200 \mathrm{~ms}$. The $200 \mathrm{~ms}$ exposure time was necessary in order to obtain a satisfactory fluorescence signal. Both particles were tethered with a 2672 bp dsDNA fragment onto a gold support as explained under Material and Methods. The FluoSpheres 
were detected using an epi-fluorescent microscope (Leica; excitation filter: band pass 340-380 nm; dichromatic mirror $400 \mathrm{~nm}$; emission filter: long pass $425 \mathrm{~nm}$ ). In view of the rapid photobleaching $(<5 \mathrm{~min})$ fluorescent particles do not seem to be appropriate for TPM measurements.

We have demonstrated that the combination of small gold particles, darkfield microscopy and a CCD camera results in a sophisticated TPM method that permits the study of dsDNA tethers under various conditions. We propose that darkfield-TPM (DFTPM) can be used for the characterization of DNA and RNA-protein interactions, protein/protein interactions, and the screening of DNA-binding proteins. This method is suitable for multiplexing - multiple non-interacting tethers in one field-of-view — in order to meet the requirement for a screening procedure. The DNA or RNA tethers would be immobilized onto a solid support at defined positions. This procedure would be comparable to DNA microarrays.

\section{Acknowledgments}

We thank Wim van Oel and Guus Liqui Lung for their technical support. We thank Prof. Dr. Hans Tanke and Prof. Dr. Simon de Vries for their support on biochemical issues. We further thank Prof. Dr. Theo Odijk for his theoretical calculation of the tethered particle's position probability distribution. We thank Sanneke Brinkers for her input on the image analysis and data fitting. We thank Bart Vermolen for his support in the use of Matlab. HAH and FGMW are financially supported by the Netherlands Organization for Scientific Research (NWO). This work was supported by the BSIK programs Cyttron and Microned (2F), and the Delft Interdisciplinary Research program (DIOC): Molecular Recognition of Biomacromolecules in Life Science and Technology.

\section{References}

[1] D. Spetzler, J. York, C. Dobbin, J. Martin, R. Ishmukhametov, L. Day, J. Yu, H. Kang, K. Porter, T. Hornung, and W. D. Frasch, "Recent developments of biomolecular motors as on-chip devices using single molecule techniques," Lab Chip 7 (12), 1633-1643 (2007) [doi:10.1039/b711066a].

[2] Y. Ishii and T. Yanagida, "Single molecule detection in life science," Single Mol. 1, 5-16 (2000) [doi:10.1002/(SICI)1438-5171(200004)1:1<5::AID-SIMO5>3.0.CO;2A].

[3] P. Hinterdorfer and Y. F. Dufrêne, "Detection and localization of single molecular recognition events using atomic force microscopy," Nat. Methods 3 (5), 347-355 (2006) [doi:10.1038/nmeth871].

[4] K. O. Greulich, "Single-molecule studies on DNA and RNA," Chem. Phys. Chem. 6, 2458-2471 (2005) [doi:10.1002/cphc.200500038].

[5] T. van der Heijden, M. Modesti, S. Hage, R. Kanaar, C. Wyman, and C. Dekker, "Homologous recombination in real time: DNA strand exchange by reca," Mol. Cell 30 (4), 530-538 (2008) [doi:10.1016/j.molcel.2008.03.010].

[6] D. A. Koster, F. Czerwinski, L. Halby, A. Crut, P. Vekhoff, K. Palle, P. B. Arimondo, and N. H. Dekker, "Single-molecule observations of topotecan-mediated topib activity at a unique DNA sequence," Nucleic Acids Res. 36 (7), 2301-2310 (2008) [doi:10.1093/nar/gkn035].

[7] U. Bockelmann, "Single-molecule manipulation of nucleic acids," Curr. Opin. Struct. Biol. 14 (3), 368-373 (2004) [doi:10.1016/j.sbi.2004.03.016].

[8] S. B. Smith, L. Finzi, and C. Bustamante, "Direct mechanical measurements of the elasticity of single DNA molecules by using magnetic beads," Science 258 (5085), 1122-1126 (1992) [doi:10.1126/science.1439819].

[9] C. Bustamante, "Ten years of tension: Single-molecule DNA mechanics," Nature 421, 423-427 (2003) [doi:10.1038/nature01405].

[10] J. F. Allemand, D. Bensimon, and V. Croquette, "Stretching DNA and RNA to probe their interactions with proteins," Curr. Opin. Struct. Biol. 13 (3), 266-274 (2003) [doi:10.1016/S0959-440X(03)00067-8]. 
[11] T. Ha, A. Y. Ting, J. Liang, W. B. Caldwell, A. A. Deniz, D. S. Chemla, P. G. Schultz, and S. Weiss, "Single-molecule fluorescence spectroscopy of enzyme conformational dynamics and cleavage mechanism," Proc. Natl. Acad. Sci. US A 96 (3), 893-898 (1999) [doi:10.1073/pnas.963.893].

[12] M. Capitanio, F. Vanzi, C. Broggio, R. Cicchi, D. Normanno, G. Romano, L. Sacconi, and F. S. Pavone, "Exploring molecular motors and switches at the singlemolecule level," Microsc. Res. Tech. 65 (4-5), 194-204 (2004) [doi:10.1002/jemt.20126].

[13] D. A. Schafer, J. Gelles, M. P. Sheetz, and R. Landick, "Transcription by single molecules of RNA polymerase observed by light microscopy," Nature 352 (6334), 444-448 (1991) [doi:10.1038/352444a0].

[14] F. Vanzi, C. Broggio, L. Sacconi, and F. S. Pavone, "Lac repressor hinge flexibility and DNA looping: Single molecule kinetics by tethered particle motion," Nucleic Acids Res. 34 (12), 3409-3420 (2006) [doi:10.1093/nar/gk1393].

[15] L. Finzi and J. Gelles, "Measurement of lactose repressor-mediated loop formation and breakdown in single DNA molecules," Science 267 (5196), 378-380 (1995) [doi:10.1126/science.7824935].

[16] S. Blumberg, A. Gajraj, M. W. Pennington, and J. C. Meiners, "Three-dimensional characterization of tethered microspheres by total internal reflection fluorescence microscopy," Biophys. J. $\quad \mathbf{8 9} \quad$ (2), $1272-1281$

(2005) [doi:10.1529/biophysj.105.061242].

[17] M. N. Lambert, E. Voecker, S. Blumberg, S. Redemann, A. Gajraj, J.-C. Meiners, and N. G. Walter, "Mg2+-induced compaction of single RNA molecules monitored by tethered particle microscopy," Biophys. J. 90, 3672-3685 (2006) [doi:10.1529/biophysj.105.067793].

[18] H. Yin, R. Landick, and J. Gelles, "Tethered particle motion method for studying transcript elongation by a single RNA polymerase molecule," Biophys. J. 67 (6), 2468-2478 (1994) [doi:10.1016/S0006-3495(94)80735-0].

[19] N. Pouget, C. Dennis, C. Turlan, M. Grigoriev, M. Chandler, and L. Salome, "Single-particle tracking for DNA tether length monitoring," Nucleic Acids Res. 32 (9), e73 (2004) [doi:10.1093/nar/gnh073].

[20] F. Vanzi, S. Vladimirov, C. R. Knudsen, Y. E. Goldman, and B. S. Cooperman, "Protein synthesis by single ribosomes," Rna 9 (10), 1174-1179 (2003) [doi:10.1261/rna.5800303].

[21] B. van den Broek, F. Vanzi, D. Normanno, F. S. Pavone, and G. J. Wuite, "Realtime observation of DNA looping dynamics of type iie restriction enzymes naei and nari," Nucleic Acids Res. 34 (1), 167-174 (2006) [doi:10.1093/nar/gkj432].

[22] J. Yguerabide and E. E. Yguerabide, "Light-scattering submicroscopic particles as highly fluorescent analogs and their use as tracer labels in clinical and biological applications," Anal. Biochem. $262 \quad$ (2), 137-156 (1998) [doi:10.1006/abio.1998.2759].

[23] V. Sandoghdar, E. Klotzsch, V. Jacobsen, A. Renn, U. Hakanson, M. Agio, I. Gerhardt, J. Seelig, and G. Wrigge, "Optical detection of very small nonfluorescent nanoparticles," Chimia 60 (11), 761-764 (2006) [doi:10.2533/chimia.2006.761].

[24] J. Zheng, C. Zhang, and R. Dickson, "Highly fluorescent, water-soluble, sizetunable gold quantum dots," Phys. Rev. Lett. 93 (7), 077402 (2004).

[25] I. Braslavsky, R. Amit, B. M. Jaffar Ali, O. Gileadi, A. Oppenheim, and J. Stavans, "Objective-type dark-field illumination for scattering from microbeads," Appl. Opt. 40 (31), 5650-5657 (2001) [doi:10.1364/AO.40.005650].

[26] G. Raschke, S. Kowarik, T. Franzl, C. Soennichsen, T. A. Klar, and J. Feldmann, "Biomolecular recognition based on single gold nanoparticle light scattering," Nano Lett. 3 (7), 935-938 (2003) [doi:10.1021/n1034223+].

[27] S. Kim, P. C. Blainey, C. M. Schroeder, and X. S. Xie, "Multiplexed singlemolecule assay for enzymatic activity on flow-stretched DNA," Nat. Methods 4 (5), 397-399 (2007). 
[28] J. Yuan and H. C. Berg, "Resurrection of the flagellar rotary motor near zero load," $\begin{array}{lllllllll}\text { Proc. Natl. Acad. Sci. } U & S & A & 105 & \text { (4), } & 1182-1185 & \text { (2008) }\end{array}$ [doi:10.1073/pnas.0711539105].

[29] M. K. Cheezum, W. F. Walker, and W. H. Guilford, "Quantitative comparison of algorithms for tracking single fluorescent particles," Biophys. J. 81 (4), 2378-2388 (2001) [doi:10.1016/S0006-3495(04)75884-5].

[30] R. J. Ober, S. Ram, and E. S. Ward, "Localization accuracy in single-molecule microscopy," Biophys. J. 86 (2), 1185-1200 (2004) [doi:10.1016/S00063495(04)74193-4].

[31] J. Yguerabide and E. E. Yguerabide, "Resonance light scattering particles as ultrasensitive labels for detection of analytes in a wide range of applications," $J$. Cell. Biochem. Suppl. Suppl 37, 71-81 (2001).

[32] K. A. Lidke, B. Rieger, D. S. Lidke, and T. M. Jovin, "The role of photon statistics in fluorescence anisotropy imaging," IEEE Tran. Image Process. 14 (9), 1237-1245 (2005) [doi:10.1109/TIP.2005852458].

[33] D. E. Segall, P. C. Nelson, and R. Phillips, "Volume-exclusion effects in tetheredparticle experiments: Bead size matters," Phys. Rev. Lett. 96 (8), 088306 (2006) [doi:10.1103/PhysRevLett.96.088306].

[34] P. J. Hagerman, "Flexibility of DNA," Annu. Rev. Biophys. Biophys. Chem. 17, 265-286 (1988) [doi:10.1146/annurev.bb.17.060188.001405].

[35] M. Rubinstein and R. H. Colby, Polymer Physics, Oxford University Press, New York (2003).

[36] O. Kratky and G. Porod, "Röntgenuntersuchung gelöster fadenmoleküle," Rec. Trav. Chim. Pays-Bas 68, 1106-1123 (1949).

[37] C. Bustamante, S. B. Smith, J. Liphardt, and D. Smith, "Single-molecule studies of DNA mechanics," Curr. Opin. Struct. Biol. 10 (3), 279-285 (2000) [doi:10.1016/S0959-440X(00)00085-3].

[38] C. Bustamante, J. F. Marko, E. D. Siggia, and S. Smith, "Entropic elasticity of lambda-phage DNA," Science $265 \quad$ (5178), 1599-1600 (1994) [doi:10.1126/science.8079175].

[39] Y. Lu, B. Weers, and N. C. Stellwagen, "DNA persistence length revisited," Biopolymers 61 (4), 261-275 (2001) [doi:10.1002/bip.10151].

[40] D. Porschke, "Structure and dynamics of double helices in solution: Modes of DNA bending," J. Biomol. Struct. Dyn. 4 (3), 373-389 (1986).

[41] C. G. Baumann, S. B. Smith, V. A. Bloomfield, and C. Bustamante, "Ionic effects on the elasticity of single DNA molecules," Proc. Natl. Acad. Sci. U S A 94 (12), 6185-6190 (1997) [doi:10.1073/pnas.94.12.6185].

[42] M. D. Wang, H. Yin, R. Landick, J. Gelles, and S. M. Block, "Stretching DNA with optical tweezers," Biophys. J. 72 (3), 1335-1346 (1997) [doi:10.1016/S00063495(97)78780-0].

[43] V. A. Bloomfield, "DNA condensation by multivalent cations," Biopolymers 44, 269-282 (1997) [doi:10.1002/(SICI)1097-0282(1997)44:3<269::AIDBIP6>3.0.CO;2T].

[44] N. E. Dorsey, Properties of Ordinary Water-Substance in All its Phases: Water Vapor, Water, and All the Ices, Reinhold Pub. Corp., New York (1940).

[45] S. Brinkers, H. R. C. Dietrich, F. H. De Grote, I. T. Young, and B. Rieger, "The persistence length of double stranded DNA determined using dark field tethered particle motion," J. Chem. Phys. 130, 215105 (2009) [doi:10.1063/1.3142699].

[46] H. M. Schessler, D. S. Karpovich, and J. G. Blanchard, "Quantitating the balance between enthalpic and entropic forces in alkanethiol/gold monolayer self assembly," J. Am. Chem. Soc. 118, 9645-9651 (1996) [doi:10.1021/ja961565r].

[47] A. Ulman, "Formation and structure of self-assembled monolayers," Chem. Rev. 96 (4), 1533-1554 (1996) [doi:10.1021/cr9502357].

[48] J. C. Love, L. A. Estroff, J. K. Kriebel, R. G. Nuzzo, and G. M. Whitesides, "Selfassembled monolayers of thiolates on metals as a form of nanotechnology," Chem. Rev. 105, 1103-1169 (2005) [doi:10.1021/cr0300789]. 
[49] D. K. Schwartz, "Mechanisms and kinetics of self-assembled monolayer formation," Аnпu. Rev. Phys. Chem. 52, 107-137 (2001) [doi:10.1146/annurev.physchem.52.1.107].

[50] F. Schreiber, "Structure and growth of self-assembling monolayers," Prog. Surf. Sci. 65, 151-256 (2000) [doi:10.1016/S0079-6816(00)00024-1].

[51] W. P. Wong and K. Halvorsen, "The effect of integration time on fluctuation measurements: Calibrating an optical trap in the presence of motion blur," Opt. Exp. 14 (25), 12517-12531 (2006) [doi:10.1364/OE.14.012517].

[52] K. B. Towles, J. F. Beausang, H. G. Garcia, R. Phillips, and P. C. Nelson, "Firstprinciples calculation of DNA looping in tethered particle experiments," submitted to: Phys Biol (2008).

[53] D. Porschke, "Persistence length and bending dynamics of DNA from electrooptical measurements at high salt concentrations," Biophys. Chem. 40 (2), 169-179 (1991) [doi:10.1016/0301-4622(91)87006-Q].

[54] T. Odijk, "Polyelectrolytes near the rod limit," J. Polym. Sci. Polym. Phys. Ed. 15, 477-483 (1977) [doi:10.1002/pol.1977.18150307].

[55] J. Skolnick and M. Fixman, "Electrostatic persistence length of a wormlike polyelectrolyte," Macromolecules 10, 944-948 (1977) [doi:10.1021/ma60059a011].

[56] M. A. Bevan and D. C. Prieve, "Hindered diffusion of colloidal particles very near to a wall: Revisited," J. Chem. Phys. 113 (3), 1228-1236 (2000) [doi:10.1063/1.481900]. 